\title{
The Study on Temperature Characteristics of a Monolithic Fused Silica
}

\section{Cylindrical Resonator}

\author{
Yunxiao $\mathrm{Wu}^{\mathrm{a}}$, Yao Pan ${ }^{\mathrm{b}^{*}}$, Dongya Wang ${ }^{\mathrm{c}}$, Tianliang Qu, Yun Huang \\ College of Optoelectronic Science and Engineering, National University of Defense Technology, \\ Changsha 410073, China
}

aemail:wyunxxd@163.com, bemail:panyao.liz@163.com, cemail: mywangdongya@126.com

Keywords: monolithic; fused silica cylindrical resonator; frequency; temperature

\begin{abstract}
Although fused silica has better temperature characteristics than most materials, temperature drift is still one of the main error sources of a Cylindrical Vibratory Gyroscope (CVG) with a monolithic fused silica resonator. The change of temperature changes the characteristics of the material, at the same time changes the overall working status of gyroscope. In this paper, the influence of temperature on the vibration characteristics of fused silica cylindrical resonator is theoretically analyzed and experimentally verified. Results show that the vibration frequency is linearly related to temperature, which provides reference for temperature compensation and performance improvement of CVG.
\end{abstract}

\section{Introduction}

Cylindrical Vibratory Gyroscope (CVG) is a new type of vibratory gyroscope, which is advantageous in not only high precision and long life, but simple structure, low cost, strong resistance to external vibration and electromagnetic interference, therefore it has broad applications in the tactical-level inertial navigation system [1]. Most reported CVGs have metal resonators, and the high loss in the material limited the quality factor to10000 40000[2,3,4,5,6]. Fused silica, on the other hand, is an ideal low-loss material. It was predicted by Innalabs that the $\mathrm{Q}$ factor of a 25 -mm diameter resonator may reach 500000 700000, and the precision of the gyroscope may achieve a few of hundredths degree per hour in the $[-40+50]{ }^{\circ} \mathrm{C}$ temperature range. However, there have been no relevant experimental results.

The temperature drift severely limits the detection accuracy and bias stability of the gyroscope. Conventional approaches for CVG temperature compensation is to utilize fuzzy algorithm and statistical methods to build models, and to use external temperature sensors to monitor the temperature [7,8]. However, for CVGs, external sensors attached on the resonator will disrupt the vibration of the resonator, at the same time induce thermal hysteresis. In contrast, utilizing the materials properties of resonator for the analysis and compensation of the temperature drift of CVGs does not have those limitations. The implementation of this method requires theoretical analysis and accurate measurement of the thermal characteristics of the resonator. Ma et. al. theoretically analyzed the influence of temperature variation on the mode frequency of a metal resonator gyroscope, then verified the analysis by simulation and experiments, based on which a compensation method was briefly mentioned [9]. As for CVGs with metallic resonators, Zhang et. al. studied the influence mechanism of temperature variation on the bias, driving voltage and mode frequency of the gyroscope, and they experimentally verified that the temperature-bias model was most effective in their case [10]. Some researchers used the relationship between the gyro output, the driving phase and temperature 
to design a new compensation algorithm without external thermal sensor, which had effectively improved the performance of a MEMS gyro [11]. The temperature variation changes the characteristics of the material, such as thermal expansion coefficient, thermal conductivity and Young's modulus, etc. In addition, it also affects the electronics and changes the overall working status of the gyroscope.

High-purity fused silica is highly isotropic and has low mechanical loss, making it the ideal material for shell resonators. Furthermore, the temperature-frequency characteristics is highly linear, therefore it is possible to utilize one mode as a measure of gyroscope temperature for the compensation of the sensing mode [12]. Our group has designed and fabricated a monolithic fused silica cylindrical resonator that has high quality factor and small frequency mismatch [13], which is expected to be applied to the high precision inertial navigation and platform stabilization systems. In this paper, the Temperature Coefficient of Frequency (TCF) was studied for this resonator. In the following sections, the structure and working principles of the monolithic fused silica cylindrical resonator are first briefly introduced. Then, the influence of temperature on the vibration characteristics of resonator is theoretically analyzed and experimentally verified. Results show that the frequency is linearly related to temperature, and the TCF of the monolithic fused silica cylindrical resonator is repeatable and stable. This proves the feasibility of using self-calibration for temperature compensation.

\section{The Structure and Working Principle}

The monolithic fused silica resonator, as shown in Figure 1, is mainly composed of a resonant shell, a vibration-conducting body (a vibration-conducting shell and a bottom plate) and an anchor. The resonator mostly vibrates at the lowest flexural mode when working as a Cylindrical Vibratory Gyroscope (CVG). The mode amplitude is detected by piezoelectric elements or by capacitive detection. As an example, the working principle of CVG based on piezoelectric effects is as follows: an $\mathrm{AC}$ voltage with a certain amplitude and frequency is applied to a pair of electrodes on the $\mathrm{Y}$ direction, so that the driving mode on the $\mathrm{X}-\mathrm{Y}$ direction is excited. Ideally, the antinode has the maximum amplitude, and the node amplitude is zero, as shown in Figure 2. When there is an angular velocity $\omega$ along the axis of symmetry, owing to the Coriolis force $F_{g}$, the energy transfers from the driving mode to the sensing mode which lies at $45^{\circ}$ relative with $X$, along the $X^{\prime}-Y^{\prime}$ direction. In the open-loop mode, the detection electrodes at $\mathrm{X}^{\prime}$ direction gives a voltage signal that is proportional to the input angular rate. In the force-to-rebalance mode, the force applied in Y' direction to suppress the sensing mode is proportional to the input angular rate.

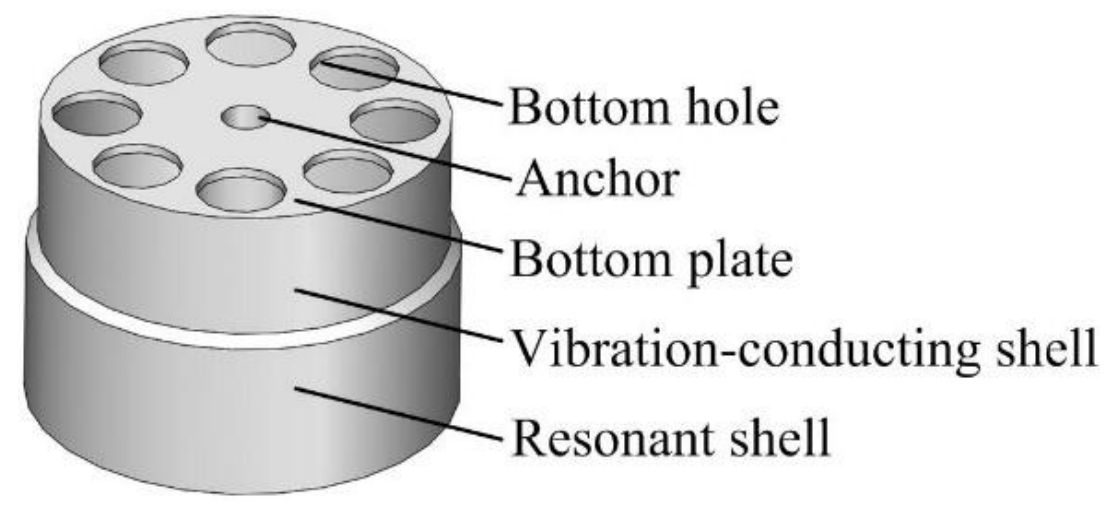

Fig. 1 The structure of the monolithic fused silica cylindrical resonator 


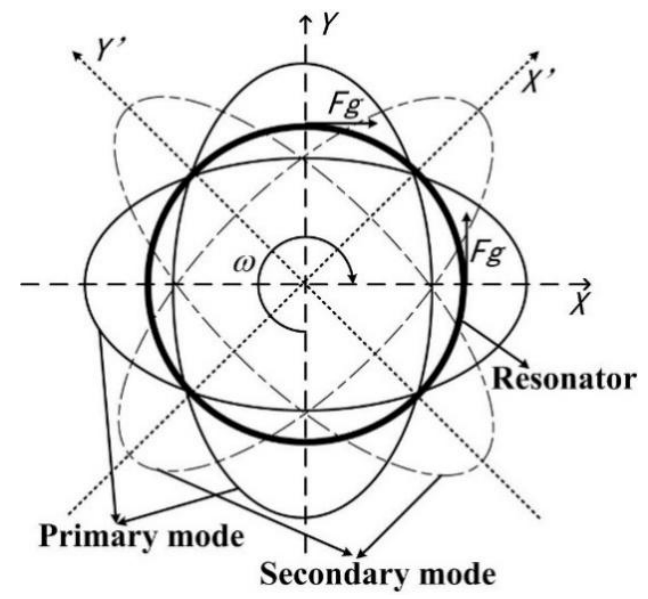

Fig. 2 The schematic of working principle of a typical CVG

\section{Theoretical Analysis}

For a fused silica resonator, when temperature changes, the elastic modulus, damping coefficient, thermal expansion coefficient and residual stress etc. change accordingly. The elastic modulus variation leads to the stiffness variation, as a result the resonance frequency of the gyroscope changes. The temperature-dependent elastic modulus of fused silica can be expressed as [14]:

$$
E(T)=E_{0}\left[1+k_{E T}\left(T-T_{0}\right)\right]
$$

Where $E(T)$, and $E_{0}$ are the elastic modulus of fused silica at temperature $T$, and $T_{0}$, respectively; $k_{E T}$ is the temperature coefficient for elastic modulus of the material.

As the system stiffness $K$ is directly proportional to the elastic modulus $E$, the variation of system stiffness caused by elastic modulus variation is as follows:

$$
\Delta K_{E}=k_{E T} K_{0}\left(T-T_{0}\right)
$$

Where $K_{0}$ is the system stiffness at temperature $T_{0}, \Delta K_{E}$ is the stiffness variation induced by elastic modulus variation.

In addition, the thermal stress changes with temperature as in:

$$
\sigma=\alpha E_{0}\left(T-T_{0}\right)
$$

Where $\alpha$ is the linear thermal expansion coefficient of fused silica.

As a result, the variation of system stiffness $\Delta K_{\sigma}$ induced by the variation of the thermal stress $\sigma$ is as follows:

$$
\Delta K_{\sigma}=\lambda_{\sigma} K_{0} \sigma=\lambda_{\sigma} K_{0} \alpha E_{0}\left(T-T_{0}\right)
$$

Where $\lambda_{\sigma}$ is thermal stress ratio which denotes the change of stiffness caused by thermal stress.

Then, the relationship between the system stiffness and temperature can be expressed as:

$$
K=K_{0}+\Delta K_{E}+\Delta K_{\sigma}=K_{0}\left[1+\left(k_{E T}+\lambda_{\sigma} \alpha E_{0}\right)\left(T-T_{0}\right)\right]
$$

Thus, the relationship between natural frequency of the resonator and temperature is [15]:

$$
\begin{aligned}
f(T) & =\sqrt{\frac{K}{m}}=\sqrt{\frac{K_{0}\left[1+\left(k_{E T}+\lambda_{\sigma} \alpha E_{0}\right)\left(T-T_{0}\right)\right]}{m}} \\
& =f_{0} \sqrt{1+\left(k_{E T}+\lambda_{\sigma} \alpha E_{0}\right)\left(T-T_{0}\right)}
\end{aligned}
$$


Where $m$ is the resonator effective mass. $f(T)$ and $f_{0}$ are the resonant frequency at temperature $T$ and $T_{0}$, respectively.

\section{Experiment}

The experimental system is composed of a temperature controlling tank, a laser Doppler vibrometer, a loudspeaker, a thermometer, and a computer, as shown in Figure 3. The resonator and the loudspeaker are fixed on a base inside the temperature controlling tank, and the vibration characteristics of resonator are measured by a laser Doppler vibrometer through the window. In addition, the temperature near the resonator is monitored by a thermometer (four probes of which are evenly located around the resonator) for comparison.

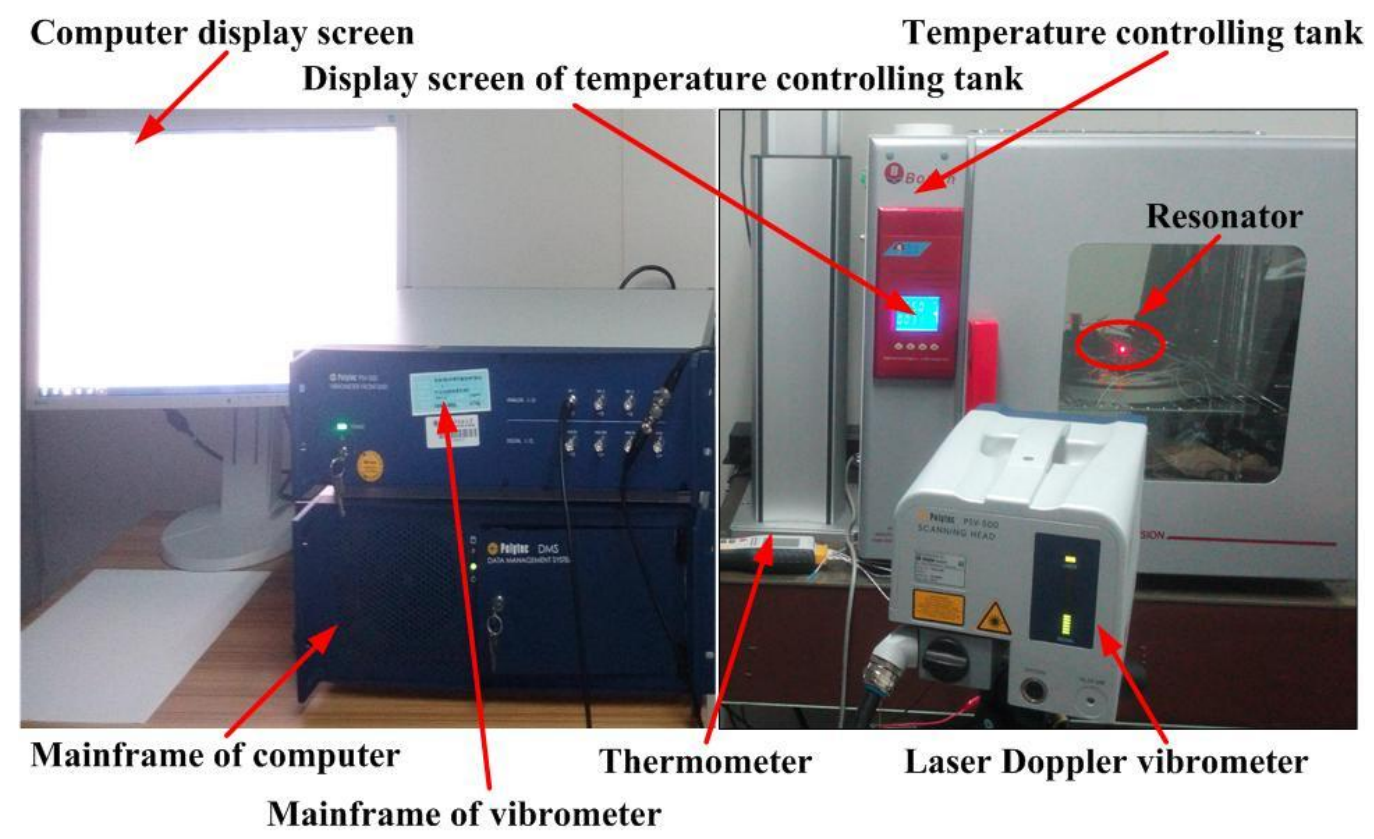

Fig. 3 The measuring system of vibration characteristics of resonator

Table 1 resonance frequency and temperature of resonator

\begin{tabular}{ccc}
\hline \multirow{2}{*}{$\begin{array}{c}\text { Temperature } \\
\left.{ }^{\circ} \mathrm{C}\right]\end{array}$} & Heating & Cooling \\
\cline { 2 - 3 } 20 & 3967.0972 & 3967.1730 \\
25 & 3968.7962 & 3968.8480 \\
30 & 3970.8836 & 3970.9470 \\
35 & 3973.0516 & 3972.9712 \\
40 & 3975.1194 & 3974.8852 \\
45 & 3977.2240 & 3977.0580 \\
50 & 3979.2432 & 3979.0356 \\
55 & 3981.2622 & 3980.9788 \\
60 & 3983.1520 & 3983.2448 \\
65 & 3985.1392 & 3985.1878 \\
70 & 3987.0482 & 3987.1194 \\
75 & 3988.9844 & 3989.0088 \\
80 & 3990.9572 & 3990.8642 \\
\hline
\end{tabular}

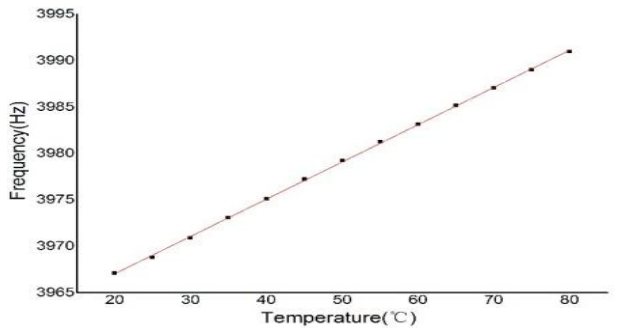

(a)

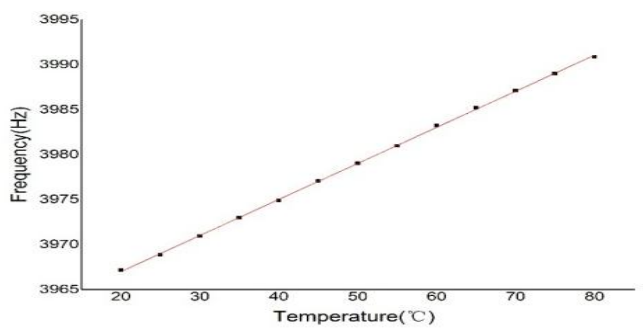

(b)

Fig. 4 Frequency-temperature measurements of (a) the heating process and (b) the cooling process 
Temperature range was set as $+20{ }^{\circ} \mathrm{C}$ to $+80{ }^{\circ} \mathrm{C}$ with a temperature increment of $5{ }^{\circ} \mathrm{C}$. By repetitive experiments we found that the resonant frequency was stable half an hour after the temperature was stable, and the temperature shown on the tank display screen was the same as the thermometer. Therefore, we measured the frequency of the resonator half an hour after the change of temperature. The resonant frequency of the monolithic fused silica cylindrical resonator was measured both in the heating and cooling process, and at each temperature point the resonant frequency was measured for five times. The averaged results were listed in Table 1.

The results were linearly fitted, as shown in Figure 4. The fitted results were as follows:

$$
\begin{aligned}
& f_{i}(T)=3959.01188+0.40124 T \\
& f_{d}(T)=3958.97362+0.40102 T
\end{aligned}
$$

Where $f_{i}(T)$ was the resonant frequency of the heating process while $f_{d}(T)$ was that of the cooling process.

Results show that the resonant frequency of the monolithic fused silica cylindrical resonator is positively correlated with temperature. The fitted temperature coefficients of frequency are $0.40124 \mathrm{~Hz} /{ }^{\circ} \mathrm{C}$ and $0.40102 \mathrm{~Hz} /{ }^{\circ} \mathrm{C}$ in the heating and cooling process, respectively. Compared with results in previous studies $\left(0.5 \mathrm{~Hz} /{ }^{\circ} \mathrm{C}\right)[16]$, these coefficients are in the same order of magnitude. The difference may be caused by differences in materials and geometric structure. The correlation coefficients of the fitting curves are 0.99966 and 0.99972, respectively, demonstrating the good linearity. In addition, the relative difference of coefficients between the heating and cooling process is approximately $549 \mathrm{ppm}$, which promises the good repeatability of the Temperature Coefficient of Frequency (TCF). This also verifies the possibility of using self-calibration based on temperaturefrequency characteristics for temperature drift compensation.

However, as can be seen from Table 1, although for most temperature points, the frequency difference between heating and cooling process was less than $0.1 \mathrm{~Hz}$, the differences from $40^{\circ} \mathrm{C}$ to $55^{\circ} \mathrm{C}$ increased to about $0.2 \mathrm{~Hz}$. The reason behind requires further investigation.

\section{Conclusions}

In this paper, the temperature-frequency characteristics of the monolithic fused silica cylindrical resonator were studied theoretically and experimentally. By measuring the resonant frequency in a temperature range of $+20^{\circ} \mathrm{C}$ to $+80^{\circ} \mathrm{C}$ during the heating and cooling process, the linear relationship between resonant frequency and temperature was demonstrated. Results showed that the monolithic fused silica resonator has a TCF of about $0.40124 \mathrm{~Hz} /{ }^{\circ} \mathrm{C}$ and $0.40102 \mathrm{~Hz} /{ }^{\circ} \mathrm{C}$ in the heating and cooling process, respectively. The small relative change showed that the temperature-frequency coefficient is repeatable and stable. This proves the feasibility of using self-calibration based on temperaturefrequency characteristics of the resonator for temperature drift compensation.

\section{References}

[1] XU Jiang-ning, BIAN Shao-feng, YIN Li-wu. Principles of Gyroscopes [M]. Bei Jing: National Defense Industry Press, 2005.

[2] Chikovani V V, Yatsenko Y A, Barabashov A S, et al. Improved accuracy metallic resonator CVG [J]. Aerospace and Electronic Systems Magazine, IEEE, 2009, 24(5):40-43.

[3] Information on http://watson-gyro.com/product/rate-gyros/pro-gyro-professional-grade-rategyro/ 
[4] Information on http://www.innalabs.com/en/products/gyroscopes/

[5] Watson W S, Henke T J. Coriolis gyro configuration effects on noise and drift performance [C]//SYMPOSIUM GYRO TECHNOLOGY. 2002: 1.1-1.1.

[6] Xi X, Wu X, Zhang Y, et al. A study on Q factor of the trimmed resonator for vibratory cupped gyroscopes [J]. Sensors and Actuators A: Physical, 2014, 218: 23-32.

[7] ZHAO Xiao-ning, LI Xian-luo, LEI Bao-quan. Temperature compensation for ring laser gyro [J]. Journal of Chinese Inertial Technology, 2004, 12 (3):55-57.

[8] FENG Li-shuang, NAN Shu-zhi, JIN Jing. Research on modeling and compensation technology for temperature errors of FOG [J]. Journal of Astronautics, 2006, 27 (5):939-941.

[9] MA Xiao-fei, SU Zhong. Research on Temperature Characteristic of Metal Resonator of Solid Wave Gyro [J]. Navigation and Control, 2015(3).

[10] ZHANG Yong-meng, WU Yu-lie, XI Xiang, et a1. Study on the temperature-dependent performance and compensation methods for cupped gyroscope [J]. Journal of Transducer Technology, 2012, 25(9):1230-1235.

[11] CHEN Huai, ZHANG Rong, ZHOU Bin, et a1. Research on thermal characteristic and compensation algorithm for MEMS-gyroscope [J]. Journal of Transducer Technology, 2004, 23(10): 24-26.

[12] X. Wang, W. Wu, Z. Fang, B. Luo, Y. Li, Q. Jiang, Temperature drift compensation for hemispherical resonator gyro based on natural frequency, Sensors 12 (5) (2012) 6434-6446.

[13] Y. Pan, D. Y. Wang, Y. Y. Wang, J. P. Liu, S. Y. Wu , T. L. Qu , K. Y. Yang, L. Hui. Monolithic cylindrical fused silica resonators with high $\mathrm{Q}$ factors. Sensors, under review.

[14] TAN Yi-yun, YU Hong, HUANG Qing-an, LIU Tong-qing. Effect of Temperature on the Young's Modulus of Silicon Nano-Films [J]. CHINESE JOURNAL OF ELECTRON DEVICES, 2007, 30(6):755 758.

[15] Xinxin Li, Takahito Ono, Yuelin Wang, et al. Study on Ultra-Thin NEMS Cantilevers-High Yield Fabrication and Size-Effect on Young's Modulus of Silicon [J]. IEEE, 2002:427 430.

[16] ZHOU Xiao-gang, WANG Li-xin, SHE Qiang, FANG Zhen, PENG Hui. HRG Temperature Compensation and Experiment Research [J]. Journal of Astronautics, 2010(4). 